\section{Expert
pinion}

1. Introduction

2. Use of RNAi

3. Angiogenesis

4. In vitro inhibition of angiogenesis using RNA

5. In vivo inhibition of angiogenesis using RNAi

6. Discussion

7. Expert opinion/ concluding remarks

\title{
siRNA-mediated inhibition of angiogenesis
}

\author{
Raymond M Schiffelers ${ }^{\dagger}$, Inge van Rooy \& Gert Storm \\ ${ }^{\dagger}$ Utrecht Institute for Pharmaceutical Sciences, Room Z 735 A, Department of Pharmaceutics, \\ Utrecht University, PO Box 80.082, 3508 TB, Utrecht, The Netherlands
}

Small interfering RNA (siRNA) has rapidly become the agent of choice for gene function analysis through loss-of-function phenotypes. Especially in complicated (patho)physiological processes such as angiogenesis, where vast numbers of proteinaceous factors are involved, the siRNA application allows relatively fast analysis of pathways and identification of new target genes. The first studies on the therapeutic effects of siRNA in angiogenesis show that this new 'drug' class holds great promise for therapeutic intervention. Two strategies emerge: the use of unmodified or the use of complexed, targeted and/or protected nucleic acids. The challenge for clinical application will be to control off-target effects and the transient character of the sequence-specific silencing effect, and to address the targeted delivery to the cell types involved in the various stages of angiogenesis. This is especially important as clinical studies indicate a profound heterogeneity of the angiogenic vasculature.

Keywords: angiogensis, siRNA

Expert Opin. Biol. Ther. (2005) 5(3):xxx-xxx

\section{Introduction}

The original observation that transgenes can induce sequence-specific messenger RNA (mRNA) degradation was made in 1990 in petunia plants on genes involved in flower pigmentation [1]. This phenomenon was termed cosuppression, as the expression of both introduced transgene and homologous endogenous gene were suppressed. In 1998, Fire et al. first described the phenomenon in an animal, the nematode Caenorhabditis elegans [2]. Injection of stretches of double-stranded RNA (dsRNA) into the worms resulted in a highly potent and specific gene silencing, which exceeded the effect of each of the single strands alone. This silencing effect was also shown to occur in Drosophila melanogaster. Kennerdell et al. [3] showed that injection of dsRNA into Drosophila embryos induced sequence-specific post-transcriptional gene silencing. This silencing effect came to be known as RNA interference (RNAi) and has now been detected in numerous eukaryotic species from the plant, fungi and animal kingdoms [4-7].

The mechanism of RNAi has not yet been completely elucidated. The system as it is now understood has been reviewed recently $[4,8,9]$ (see also Figure 1). The dsRNA trigger for the silencing machinery is cleaved to produce small interfering RNA (siRNA). siRNAs are double-stranded duplexes with two-nucleotide $3^{\prime}$ overhangs and 5'-phosphate termini. siRNA joins an effector nuclease complex, named RNA-induced silencing complex (RISC), and guides that complex to homologous mRNA. The siRNA unwinds and the RISC becomes activated. Subsequently, the mRNA is cleaved $\sim 12$ nucleotides from the $3^{\prime}$ terminus of the complementary siRNA strand [9-12]. Finally, the cleaved mRNA is probably degraded by an exoribonuclease. This sequence of events takes place in the cytosol.

Recently, siRNA was also shown to induce transcriptional gene silencing in the nucleus [13,14]. Sequence-specific methylation of histone $\mathrm{H} 3$ lysine 9 or $\mathrm{CpG}$ islands 


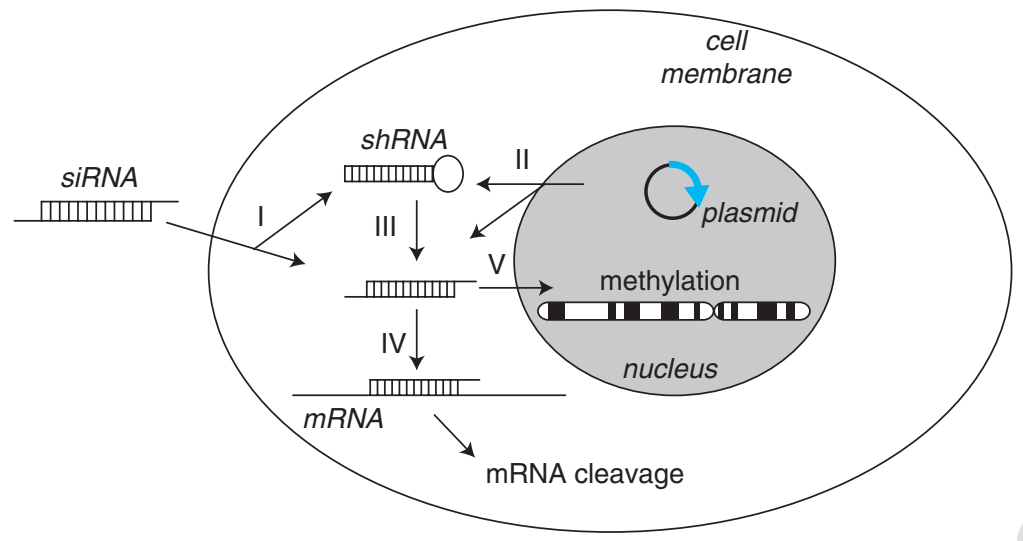

Figure 1. siRNA or shRNA can be synthesised exogenously and subsequently cells can be transfected (I). Alternatively, siRNA or shRNA may be synthesised endogenously (e.g., after transfection with si/shRNA-encoding plasmid DNA) (II). In the cytosol, shRNA is processed into siRNA (III). siRNA can enter two pathways leading to post-transcriptional or transcriptional gene silencing via mRNA cleavage (IV) or DNA methylation/histone methylation $(\mathbf{V})$, respectively.

shRNA: Short hairpin RNA; siRNA: Small interfering RNA.

in DNA promoter sequences were shown to inhibit transcription and, consequently, protein expression. As demethylation does not occur readily inside the nucleus, this process may offer a prolonged gene silencing effect.

In mammalian cells, introduction of dsRNA leads to activation of the dsRNA-dependent protein kinase (PKR) response [15]. This response induces a non-sequence-specific shutdown of protein production. However, introduction of siRNA into the cell mostly avoids activation of this pathway by virtue of its small size, thereby allowing sequence-specific silencing to occur. It must be noted that recent reports indicate that this size cutoff between nonspecific and specific gene silencing is not absolute and siRNA may also trigger pathways beyond the sequence-specific gene silencing, reminiscent of the activation of the PKR response [16-18].

Interesting aspects of RNAi in C. elegans are its potency and the ability of the effect to spread throughout the organism, even when triggered by minute quantities of dsRNA [19-21]. Several features of the RNAi mechanism have been proposed to contribute to the remarkable potency of the reaction. Some degree of amplification is likely to derive from cleavage of the dsRNA to siRNAs. For the most commonly used dsRNA of $500-1000$ bp this would result in a 20 - to 40 -fold increase in the molar ratio of trigger to target. A simple (single-use) utilisation of the siRNAs would be sufficient to explain the efficiency of RNAi in extracts of D. melanogaster on a molar basis, but would be insufficient to account for in vivo potency in C. elegans. A multi-round mechanism (use of a single siRNA for hundreds or thousands of rounds of target degradation) would be much more efficient and is a possible explanation for the amplification.

Another explanation comes from Sijen et al. [22]. According to them, the siRNAs have an appropriate structure to allow interaction in vivo with complementary sequences on the target mRNA. Two possible routes may follow this initial interaction. The first possible consequence is that the siRNA might prime the synthesis of additional dsRNA, using the target mRNA as a template, in a reaction catalysed by an RNA-directed RNA polymerase (RdRP). The newly synthesised dsRNA would then be cleaved by Dicer to generate secondary siRNAs at a sufficient concentration to achieve efficient target mRNA degradation. The second possible consequence is that cleavage of the target mRNA in the region of siRNA homology might produce an end structure that signals RdRP to initiate de novo synthesis of antisense RNA on the cleaved mRNA template.

How siRNA can leave the cell in which it was generated to enter a new target cell and silence genes is at present unknown. A receptor called SID- 1 has been postulated to bind and internalise dsRNA and may play a role $[20,21,23]$. Whether SID-1 protein has functional mammalian homologues is unclear.

This review outlines recent developments in the use of RNAi in angiogenesis for pathway analysis, target validation and therapeutic intervention.

\section{Use of RNAi}

RNAi is widely used to study gene function, especially in C. elegans [24,25]. When working with worms, introducing the dsRNA is relatively easy: dsRNA can be injected directly into the intestine of the animal, or the worm can be fed with Escherichia coli expressing the target gene-specific dsRNA $[2,26,27]$. The dsRNA has been found to inhibit expression of the target gene in different tissue types. Furthermore, the silencing effect is frequently inherited by the progeny of the injected animal. As the entire genome of C. elegans has been sequenced, RNAi is being used to help assign functions to the entire array of worm genes. This 
information may help identify pathways affected by the target gene and their relevance in diseases.

The introduction of siRNA into the cytosol of in vitro cultured mammalian cells is comparatively simple by transfecting cells with electroporated or cation-complexed siRNA, or transfection of cells with electroporated, virally delivered or cation-complexed DNA encoding siRNA or short hairpin RNA (shRNA) (Figure 2). The information obtained from the phenotypes resulting from the silencing effects may be used to assign gene functions and to validate drug targets [28-30].

The therapeutic promise of siRNA has stimulated research on a variety of diseases where (over)expression of specific genes contributes to the pathological process [31-33]. In addition, in angiogenesis, proteinaceous factors are important contributors to the switch from a quiescent to a pro-angiogenic phenotype of the vasculature and constitute possible targets for gene silencing $[34,35]$. However, therapeutic application requires the siRNA, either DNA-encoded or chemically synthesised, to be delivered to the correct cell type at the right time for achieving the desired effects. The need for temporospatial targeted delivery has proven to be a formidable barrier [36-38]. In the next section a short introduction to angiogenesis is provided, followed by an overview of the approaches taken to examine angiogenic pathways, validate new targets, or to inhibit angiogenesis by targeting siRNA to endothelial cells (ECs) or cells that support formation of the new vasculature in vivo.

\section{Angiogenesis}

Angiogenesis, the formation of new blood vessels from preexisting vasculature, plays an important role in a variety of pathological processes, such as tumour growth, inflammation, diabetic retinopathy and age-related macular degeneration [39-43]. In general, angiogenesis is the result of a local overproduction of pro-angiogenic factors shifting the balance between anti- and pro-angiogenic factors in the direction of a pro-angiogenic state [44]. This switch changes the normal quiescent vasculature, where antiangiogenic factors predominate, to an actively proliferating vasculature infiltrating host tissues. During angiogenesis one or more of the following phases can be distinguished depending on the disease type and stage.

Diseased or injured tissues produce and release pro-angiogenic growth factors (such as vascular endothelial growth factor [VEGF] and basic fibroblast growth factor [bFGF]), which diffuse into surrounding tissues. The angiogenic growth factors bind to specific receptors (such as VEGF receptor [VEGFR]2 and fibroblast growth factor-receptor) located on the surface of ECs of the pre-existing blood vessels in these tissues [45].

As a result of the binding of pro-angiogenic factors to EC surface receptors, ECs become activated. This activation leads to the production and secretion of several enzymes (such as progelatinase A) that dissolve the basement membrane. Activation also mediates changes in the coagulation pathways, such as the exposure of tissue factors on the EC surface leading to a local prothrombotic state [46].
Subsequently, the ECs start to proliferate and migrate through the basal membrane towards the site where the pro-angiogenic factors are produced by using surface proteins such as adhesion molecules and integrins (e.g., $\alpha_{v} \beta_{3}$ and $\alpha_{v} \beta_{5}$-integrin) [47].

Additional extracellular matrix-degrading enzymes, such as (membrane-bound) matrix metalloproteases (MMPs), are produced to assist the sprouting tip of the vessels. ECs close to form a lumen and these individual small tubes connect to form functional blood vessels [48].

Finally, these newly formed blood vessels are stabilised by the infiltration of specialised cells (smooth muscle cells, pericytes) that provide structural support to the new capillary [49].

In addition to proliferation of blood vessels, the tumourassociated lymphatic vessels may also grow and contribute to tumour progression and metastasis [50,51]. Recently, a number of lymphangiogenic growth factors (such as VEGF-C and -D) were characterised. These factors bind to a specific receptor on lymphatic ECs, named VEGFR3 [52]. Identification of these and other growth factors provide understanding on the molecular mechanisms and importance of lymphangiogenesis in tumour growth. As such, lymphangiogenesis opens up a new strategy for alternative treatment of tumours where inhibition of blood vessel growth is insufficient to achieve successful tumour therapy.

Taken together, both during angiogenesis and lymphangiogenesis many proteinaceous (growth) factors play a crucial role and constitute possible targets for therapeutic intervention by RNAi to inhibit these processes.

\section{In vitro inhibition of angiogenesis using RNAi}

\subsection{Pathway analysis}

Several studies have used siRNA as a tool to dissect pathways in angiogenesis. Shu et al. used siRNA to dissect the stimulatory pathway of VEGF on DNA synthesis. By silencing sphingosine kinase, VEGF was unable to induce phosphorylated extracellular signal-regulated kinase (ERK) and Ras-GTP, identifying these proteins as sequential steps in the VEGF pathway that ultimately lead to the induction of DNA synthesis [53].

Petrovic et al. investigated the downstream inductors of aminopeptidase $\mathrm{N}$ expression on the surface of activated ECs. The nuclear effector of this induction appeared to be Ets-2. siRNA-mediated inactivation of Ets-2 prevented aminopeptidase $\mathrm{N}$ expression on stimulated ECs. Furthermore, ECs stimulated to form capillaries,failed to do so when cells contained siRNA silencing Ets-2, underlining the important role of Ets-2 in angiogenesis [54].

Hypoxia-inducible factors (HIFs) play a central role in the response of ECs and cancer cells to hypoxia, and the transcription of many genes involved in angiogenesis depends on these factors. By using siRNA as a highly specific inhibitor, the role of HIF-1 could be distinguished from the role of HIF-2. Interestingly, whereas ECs and breast carcinoma cells were dependent 
1. Nucleic acid

2. Delivery
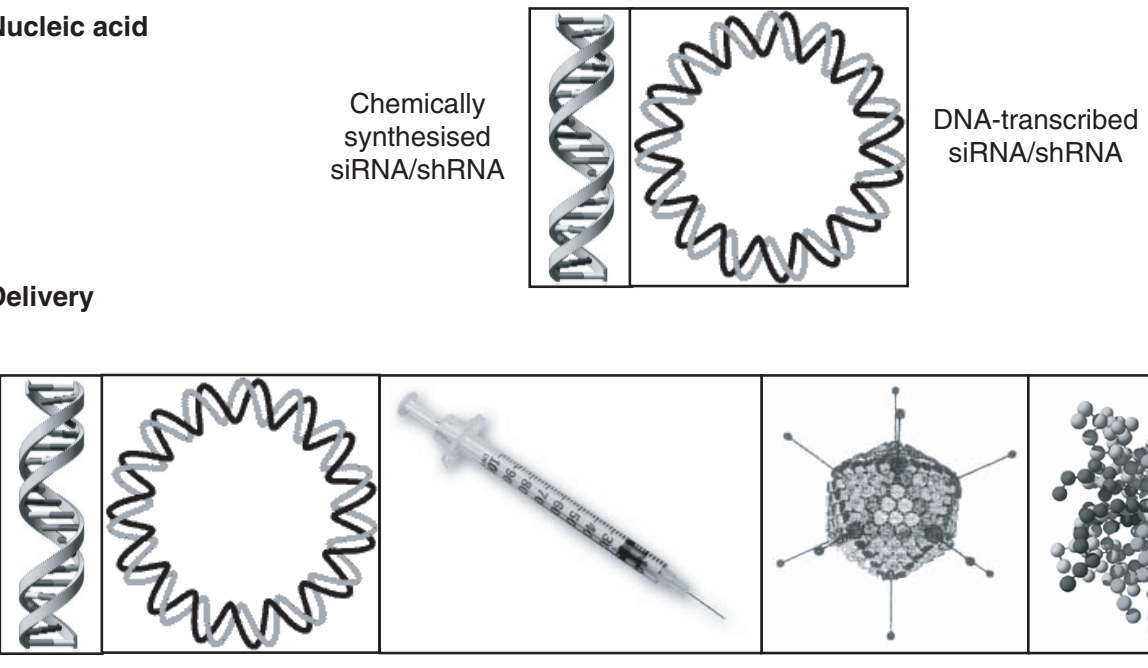

Unmodified delivery 'Naked' nucleic acids

Physical delivery
Micro-injection, electroporation, etc.

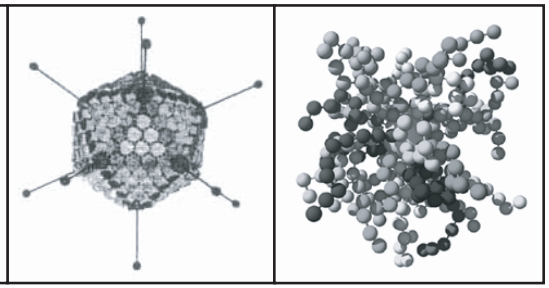

Viral vectors

Non-viral vectors Cationic complexing agents

3. In vitro

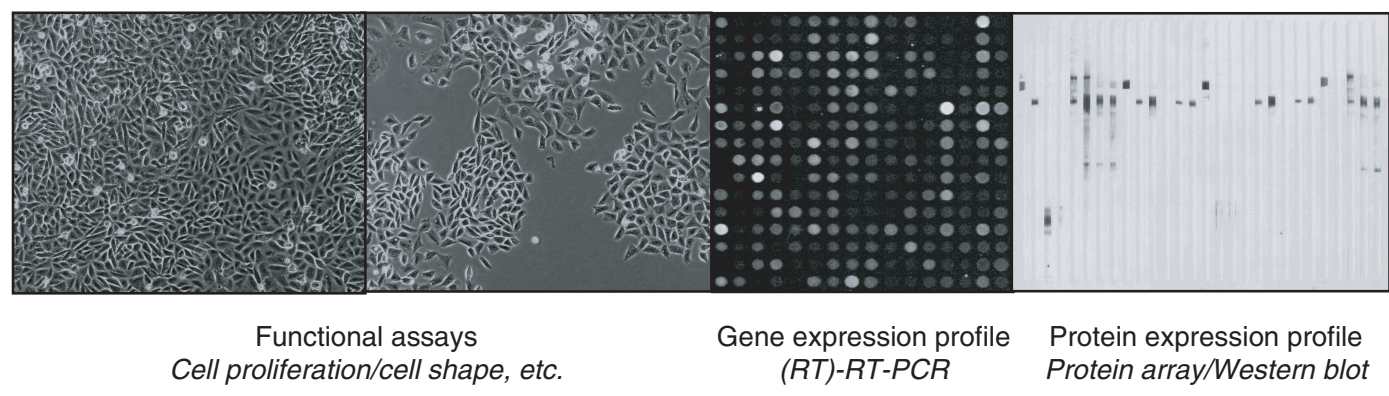

4. In vivo

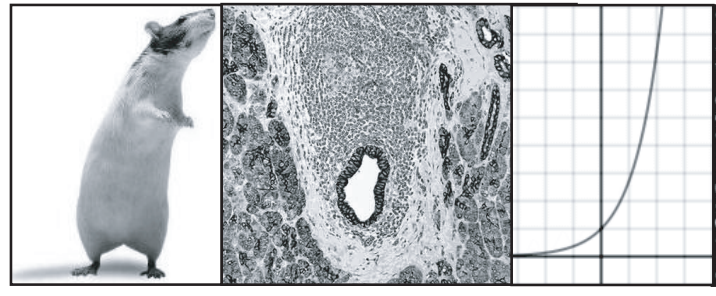

Animal experiments Tumour growth, histology, behaviour
(RT)-RT-PCR

Protein expression profile
Protein array/Western blot

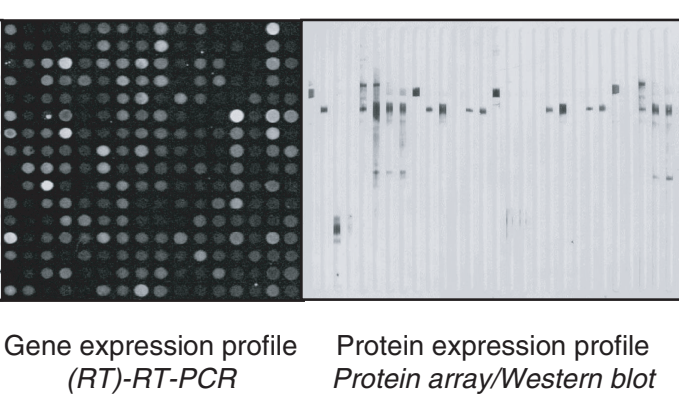

Figure 2. RNAi in angiogenesis research. 1. siRNA or shRNA can be administered as chemically synthesised nucleic acid or transcribed from encoding DNA. 2. The delivery method should be chosen depending on the application. The nucleic acids can be administered as unmodified 'naked' molecules, delivered by physical methods (such as microinjection or electroporation), delivered by viral vectors (such as adenoviruses, lentiviruses or adeno-associated viruses), or delivered by non-viral vectors (such as polycationic polymers or cationic lipids). 3. In vitro, effects of siRNA can be observed on the cellular level by investigating parameters such as cell shape and cell proliferation, and at the molecular level by determining gene expression profiles and protein expression profiles using techniques such as (RT)-RT-PCR, microarrays, protein arrays and western blotting. 4. In vivo, effects of siRNA can be observed on the level of animal or organ by investigating parameters such as behaviour, tumour growth and histology. These data can be supplemented by parameters measuring effects at the molecular level by determining gene expression profiles and protein expression profiles.

RNAi: RNA interference; (RT): Real time; RT-PCR: Reverse transcription polymerase chain reaction; shRNA: Short hairpin RNA; siRNA: Small interfering RNA. 
largely on HIF-1 for gene expression and cell migration, renal carcinoma cells activated transcription through HIF-2. This study indicates the advantage of having a highly specific inhibitor for pathway analysis to allow discrimination between two very similar factors, which may be very difficult to achieve with classical small molecular weight antagonists [55].

This advantage is also recognised in a study by Zhang et al. [56]. They developed an adenoviral vector for delivery of shRNA to knockdown specific isoforms of VEGF. As there is reason to believe that the (more than) five isoforms of VEGF have different functionalities, this tool may lead to important new insights into the biological role of each of the different VEGF-species.

The effects of two known antiangiogenic compounds, endostatin and fumagillin, were studied by Mazzanti et al. [57]. Using differential gene expression analysis they identified three genes (DOC1, TC1 and KLF4) whose expression was affected by both endostatin and fumagillin treatment. It appeared that TC1 and KLF4 expression were not upregulated in response to endostatin treatment after silencing of DOC1. After treatment with fumagillin, however, DOC1 silencing did not affect TC1 and KLF4 upregulation. These results indicate a role for DOC1 upstream from KLF4 and TC1 in angiogenic effects mediated by endostatin, whereas for fumagillin, these genes may be involved, but seem to act through an alternative signalling pathway.

As indicated in section 4, one of the important steps during angiogenesis is the migration of ECs and infiltration into the tissue. By knocking down the production of junctional adhesion molecule-1, Naik et al. were able to show that human umbilical vein ECs (HUVEC) stimulated with bFGF were no longer able to spread and migrate onto a vitronectin-coated surface, supporting an important role for this adhesion molecule in EC migration [58]. Next to that, silencing of junctional adhesion molecule-1 in ECs also prevented activation of the ERK-pathway normally induced by bFGF treatment.

These examples illustrate the use of RNAi in exploring pathways and mediators that play a role in the angiogenic cascade. The rapid generation of potent and highly specific inhibitors for the study of loss-of-function phenotypes is an important advantage of this approach, especially when trying to separate the role of two structurally similar proteins or isoforms.

\subsection{New targets}

The method of RNAi may also be used to identify novel target proteins involved in the process of angiogenesis that may be useful for therapeutic intervention.

Sphingosine 1-phosphate phosphatase (S-1-PP) was studied by Johnson et al. [59]. Knockdown of S-1-PP by siRNA reduced mRNA levels of the enzyme and led to a twofold accumulation of S-1-P, as the phosphate of this lipid is no longer cleaved, together with a concomitant twofold reduction in unphosphorylated sphingosine levels. As S-1-P has been identified to play a functional role in the stimulation of angiogenesis, modulation of the activity of S-1-PP may constitute a therapeutic target in angiogenesis.

Sullivan et al. identified a novel protein disulfide isomerase preferentially expressed in tumour ECs, named endo-PDI [60]. Normal PDI acted as a survival factor for ECs in situations with normal oxygen pressure and under hypoxic conditions. By using siRNA they were able to demonstrate that endo-PDI only did so under hypoxia. As inhibition of endo-PDI resulted in concomitant loss of proteins involved in the prevention of endothelial apoptosis, endo-PDI may represent a novel antiangiogenic target.

Diacylglycerol kinase-alpha knockdown was demonstrated to be essential for VEGF stimulation in order to achieve migration and proliferation responses of ECs [61]. Next to other inhibitors, siRNA was used to inhibit diacylglycerol kinase-alpha in HUVEC. In a Matrigel assay, HUVEC treated with diacylglycerol kinase-alpha-specific siRNA displayed strongly reduced angiogenic responses as compared with control-treated cells, indicating that inhibition of the enzyme offers prospects for inhibition of angiogenesis.

In a study to determine the functional role of homeobox proteins (Hox), inhibition of HoxA9 expression decreased EC tube formation and migration. Immunoprecipitation indicated that HoxA9 interacted with the EphB4 receptor, and that expression of the receptor promoted migratory and tube formation activity of ECs, implying that EphB4 may be a novel target for therapeutic intervention in angiogenesis [62].

The role of desmoplakin was investigated by Zhou et al. [63]. ECs stimulated to form capillaries on Matrigel were treated with siRNA silencing desmoplakin. Analysis of video recordings of these stimulated cells showed that although desmoplakin siRNA-treated cells continued to migrate towards each other and engaged in close contact, cells rarely adhered to one another or arranged themselves into elongated tubes, indicating that desmoplakin may be an interesting target to prevent formation of functional blood vessels.

These studies illustrate the attractiveness and potency of using RNAi to identify novel target proteins that can be exploited to inhibit angiogenesis. At present, there is a strong focus for increasing throughput of the technique for the screening of tens of thousands of potential drug targets per day [30,64-66].

\section{In vivo inhibition of angiogenesis using RNAi}

\subsection{Unmodified nucleic acids}

VEGF is a growth factor that promotes blood vessel formation in early embryonic development (vasculogenesis) and has a central role in angiogenesis [39,67-69]. There is compelling evidence indicating that uncontrolled angiogenesis is a major contributing factor in both tumour growth and metastasis. Inhibition of VEGF activity or disabling the function of its receptors has been shown to inhibit both tumour growth and metastasis in a variety of animal tumour models [70-72]. This 
Table 1. In vivo studies with siRNA targeting angiogenesis.

\begin{tabular}{llll}
\hline Target gene & siRNA & Route of delivery & Reference \\
\hline VEGF & 'naked' & $\begin{array}{l}\text { intramuscular, intraperitoneal, } \\
\text { intravenous, subcutaneous }\end{array}$ & 77 \\
CEACAM6 & 'naked' & intravenous & 78 \\
CSF-1 (receptor) & 'naked' & intratumoural & 79 \\
MMP-9/Cathepsin-B & plasmid-encoded & brain infusion & 80 \\
S1P1 & complexed with cationic liposomes & intratumoural & 81 \\
VEGF & complexed with atellocollagen & intratumoural & 82 \\
VEGFNEGFR1NEGFR2 & complexed with targeted cationic polymer & intravenous, local delivery in the eye \\
\hline
\end{tabular}

The table shows the target genes, delivery form of siRNA, route of administration and reference number of studies using siRNA to inhibit angiogenesis in vivo. CEACAM: Carcinoembryonic antigen-related cell adhesion molecule; CSF: Colony-stimulating factor; MMP: Matrix metalloprotease; S1P1: Sphingosine 1-phosphate receptor 1; siRNA: Small interfering RNA; VEGF: Vascular endothelial growth factor; VEGFR: VEGF receptor.

means that mediators of the VEGF-pathway could be excellent targets for RNAi in antiangiogenic therapy.

Filleur et al. conducted experiments in which VEGF was silenced to investigate the antivascular effect of RNAi (Table 1) [73].

Rat fibrosarcoma cJ4 cells were transfected with either siRNA specific for VEGF or control siRNA and grown in nude mice, and tumour growth was monitored. By day 12, immunohistochemical staining for VEGF in tumours showed a marked reduction in VEGF expression of tumours transfected with VEGF-siRNA as compared with controls. This reduction was accompanied by a $67 \%$ reduction in tumour volume. These data indicate that the reduction in VEGF synthesis obtained by the siRNA transfection in vitro resulted in the expected biological effects on VEGF production and tumour growth in vivo. The tumour growth was unaffected by the transfection of control siRNA.

In this study, siRNA was used unprotected. For in vivo applications the delivery step is of crucial importance. Therefore, many investigators employ protecting chemical modifications or complexing cations to protect and target the siRNA molecules. In the study by Filleur et al., the delivery of unmodified siRNA to tumours by various administration routes was monitored. It appeared that intraperitoneal administration of $50 \mu \mathrm{l} 125 \mu \mathrm{g} / \mathrm{kg}$ siRNA given daily gave the best knockdown effect, but also intravenous and subcutaneous injections resulted in silencing effects. Contra-intuitively, direct intratumoural injection failed to induce silencing effects. These experiments suggest that efficient delivery of siRNA into tumours via frequent parenteral administration is feasible, and showed that VEGF expression and tumour growth can be inhibited with the use of siRNA. No gross side effects were detected in the animals at the end of the experiment.

Systemic administration of unmodified siRNA was also used by Duxbury et al. They used siRNA to silence carcinoembryonic antigen-related cell adhesion molecule (CEACAM)6, which is overexpressed in a variety of gastrointestinal malignancies. siRNA was administered twice-weekly at a dose of $150 \mu \mathrm{g} / \mathrm{kg}$ via the tail vein in mice bearing BxPC3 tumours.
CEACAM6-specific siRNA was administered when tumours reached a size of $50 \mathrm{~mm}^{3}$. Tumours treated with siRNA against CEACAM6 were $68 \%$ smaller and the formation of liver metastases was prevented. CD34 staining, as a marker for progression of angiogenesis, was sevenfold lower for tumours treated with CEACAM siRNA. Although the authors realise that the efficacy of 'naked' siRNA after intravenous injection is difficult to comprehend, they suggest that the locally enhanced permeability in the tumour contributes to the observed effects [74].

Local intratumoural injection of siRNA was used in a study by Aharinejad et al. Silencing colony-stimulating factor-1 (CSF-1) or its receptor was hypothesised to reduce recruitment of tumour-associated macrophages with concomitant reduction in local production of VEGF by macrophages, thereby reducing tumour growth rate [75]. Nude mice bearing MCF-7 mammary carcinoma cells were treated with siRNA silencing CSF-1 or CSF-1 receptor with five intratumoural injections of $10 \mu \mathrm{g}$. Histomorphometric analysis of mammary tumours showed a decreased density of proliferating ECs with siRNA-mediated silencing of CSF-1 and CSF-1 receptor. In addition, mRNA levels of VEGF were reduced, but levels of mRNA for VEGFR1 or VEGFR2 remained constant.

In a study using DNA encoding shRNA, Lakka et al. silenced MMP-9 and cathepsin B [76]. In a number of in vitro angiogenesis studies, silencing of MMP-9 and cathepsin B was shown to inhibit angiogenic responses of ECs. Moreover, silencing both proteases induced stronger inhibitor effects than silencing each of the enzymes alone. In a therapeutic setting, mice bearing established SNB19 tumour cells intracranially were treated with the plasmid vector. Plasmid was infused in the brain using osmotic minipumps at a rate of $0.25 \mu \mathrm{l} / \mathrm{h}$ and a total dose of $150 \mu \mathrm{g}$. Inhibiting each of the enzymes alone induced regression of tumours to $\sim 50 \%$ of the original tumour volume. More importantly, the combination of silencing both enzymes eliminated the tumour completely. Interestingly, the plasmid vector was also applied intraperitoneally and still induced complete eradication of the tumour. This indicates that distant production of shRNA may still lead to therapeutic effects in the brain. 


\subsection{Complexed siRNA}

Sphingosine 1-phosphate receptor 1 (S1P1) is implicated in the stabilisation of newly formed vasculature in embryos. Its overexpression has also been demonstrated in tumour vessels [77]. S1P1 was targeted using multiple siRNAs specific for different regions of the mRNA transcript. Cationic liposomes based on 1,2-dioleoyl-3-trimethylammonium-propane (DOTAP) were used to complex siRNA and to augment cell interactions. siRNA silencing S1P1 inhibited EC migration in vitro and infiltration of Matrigel in vivo. In mice bearing Lewis lung carcinoma tumours, siRNA complexed to cationic liposomes was injected every third day in a volume of $60 \mu \mathrm{l}$ containing $15 \mu \mathrm{g}$ siRNA. Tumours exhibited an $\sim 50 \%$ reduction in tumour growth rate with reduction in vascular density, quantified by CD31 staining. Furthermore, the morphology of blood vessels was changed.

VEGF silencing in vivo was studied by Takei et al. [78]. They used siRNA which suppressed the synthesis of VEGF in the human prostate carcinoma cell line PC-3. PC-3 cells were injected into the flank of nude mice. After 3 weeks, visible tumours had developed at the injection sites. To determine the effectiveness of VEGF siRNA, mice were treated intratumourally with VEGF siRNAs complexed to atelocollagen, a biomaterial that is expected to increase cellular uptake and nuclease resistance and to provide prolonged release of the siRNA. Intratumoural treatment was repeated every 10 days for four rounds. VEGF siRNA markedly suppressed tumour growth compared with scrambled control siRNA or atelocollagen alone. The growth inhibitory effects were dependent on the dose of the VEGF siRNA. No gross adverse effects, for example, loss of body weight, were observed during the experiments.

\subsection{Targeted complexed siRNA}

The authors' own studies focused on the use of targeted complexing agents as carriers for siRNA for intravenous administration and cell type-specific targeted delivery [79]. Poly(ethyleneimine) (PEI) was chosen as a polycationic complexing agent, polyethylene glycol (PEG) for steric stabilisation, and a peptide ligand containing an Arg-Gly-Asp (RGD)-motif to provide selectivity by virtue of their binding to $\alpha_{\mathrm{v}}$-integrins on activated ECs. EC interaction of RGD-PEG-PEI-complexed siRNA was mediated by the RGD-peptide, as binding of nanoparticles in the presence of an excess of free peptide was $>90 \%$ reduced. Intravenous injection of RGD-PEG-PEI in mice was not associated with apparent toxicity.

Studies in vivo, in N2A-tumour bearing mice, showed that siRNA targeting murine VEGFR2 resulted in strong inhibition of tumour growth rate, which suggests uptake by activated ECs. Mice treated intravenously with $40 \mu \mathrm{g}$ siRNA silencing VEGFR2 every 3 days experienced a reduced tumour growth rate that was paralleled by a reduction in functional blood vessels in the vicinity of the tumour, supporting an antiangiogenic activity of RGD-PEG-PEI-complexed siRNA against VEGFR2.
These observations were expanded in mice suffering from herpes simplex virus DNA-induced stromal keratitis leading to angiogenesis in the eye [80]. siRNAs targeted against VEGF, VEGFR1 or VEGFR2 were able to inhibit the formation of new vasulature; however, effects were maximal when siRNAs against VEGF and both receptors were mixed. Local administration of $10 \mu \mathrm{g}$ siRNA into the conjunctiva was observed to result in the best therapeutic effect, but intravenous administration of $40 \mu \mathrm{g}$ siRNA, especially when delivered with RGD-PEG-PEI, was almost as effective. The use of a combination of siRNAs targeting various mediators in a specific pathway in the angiogenic cascade seems a highly promising approach to control ocular neovascularisation.

\section{Discussion}

\subsection{Specificity}

As mentioned in section 1 , the introduction of siRNA into cells mostly avoids activation of the PKR response, but recent reports indicate that certain siRNAs may also trigger PKR-like pathways [16-18]. In addition, certain reports question the (absolute) specificity of the siRNA approach [81-84]. The original claim that siRNA is able to distinguish between single nucleotide differences does not always hold true. It appears that the design of siRNAs should be improved. Many researchers base their sequence selection on BLAST analysis, which may lead to the selection of sequences that share substantial homology to non-target sequences [83]. Improvements in bioinformatic analysis of sequences may avoid these off-target effects. Validating each sequence for specificity on mRNA and protein levels seems important in relating mRNA downregulation and phenotypes to RNAi.

\subsection{Transient character}

One of the features of RNAi in mammalian cells is the transient character of siRNA-induced gene silencing. As opposed to worms and plants, mammals are thought to lack mechanisms that amplify silencing and ensure spreading. RNAimediated gene suppression after siRNA administration is usually observed only for $24-96 \mathrm{~h}$. To circumvent this problem, several plasmid and viral vector systems have been developed that maintain production of siRNAs/shRNAs in situ for prolonged periods of time [30,85-87]. Disadvantages of the plasmid and vector systems are the loss of control over the dose of siRNA that cells receive, the increased difficulty of functional delivery of the far larger DNA encoding siRNA/shRNA, and safety and cell specificity issues connected with the use of viruses. Another way to circumvent the transient character of siRNA treatment may be by increasing the stability of siRNA by chemical modifications [88-90].

\subsection{Targeting}

For the siRNAs to be effective, they must enter target cells. As siRNAs are relatively large molecules that are strongly negatively charged, spontaneous diffusion over the cell 
membrane is expected to be very low. Nevertheless, as described in section 6.1, silencing has been observed after simple injection of unmodified siRNAs via local or systemic routes. It remains unclear whether specific receptors (SID-1 analogues) exist that would enable the uptake of siRNA into cells or other mechanisms by which siRNA could pass the lipid bilayer. Otherwise, these results are sometimes difficult to interpret as there seems to be no relationship between the gene silencing effect and cell exposure to the silencing siRNA [73].

\section{Expert opinion/concluding remarks}

The RNAi process provides a potent method for dissecting pathways in complex pathological conditions, such as the angiogenic cascade, and for the identification of novel target proteins for use in therapeutic intervention. The method is especially important in view of its specificity and possibilities for high-throughput applications. For therapeutic applications, the remarkable efficiency of RNAi and the possibility of combining siRNAs to enhance silencing effects are attractive features. The increased recognition of the importance of RNAi since the discovery of siRNA, combined with observations on the siRNA-mediated inhibition of angiogenesis in animal models, indicates a bright future for therapeutic applications of siRNA. Challenges for the future will be to address the profound heterogeneity of ECs, thereby expanding therapeutic intervention for siRNA to the different phases of angiogenesis and corresponding phase-specific target proteins that are encountered in clinical situations.

\section{Bibliography}

Papers of special note have been highlighted as either of interest $(\bullet)$ or of considerable interest $(\bullet)$ to readers.

1. NAPOLI C, LEMIEUX C, JORGENSEN R: Introduction of a chimeric chalcone synthase gene into petunia results in reversible co-suppression of homologous genes in trans. Plant Cell (1990) 2(4):279-289.

2. FIRE A, XU S, MONTGOMERY MK et al: : Potent and specific genetic interference by double-stranded RNA in Caenorhabditis elegans. Nature (1998) 391(6669):806-811.

-. Landmark paper on the use of RNAi in animals.

3. KENNERDELL JR, CARTHEW RW: Heritable gene silencing in Drosophila using double-stranded RNA. Nat. Biotechnol. (2000) 18(8):896-898.

4. AGRAWAL N, DASARADHI PV, MOHMMED A et al.: RNA interference: biology, mechanism, and applications. Microbiol. Mol. Biol. Rev. (2003) 67(4):657-685.

5. ARENZ C, SCHEPERS U: RNA interference: from an ancient mechanism to a state of the art therapeutic application? Naturwissenschaften (2003) 90(8):345-359.

6. SCHERR M, MORGAN MA, EDER M:

Gene silencing mediated by small interfering RNAs in mammalian cells. Curr. Med. Chem. (2003) 10(3):245-256.

7. SUSI P, HOHKURI M, WAHLROOS T, KILBY NJ: Characteristics of RNA silencing in plants: similarities and differences across kingdoms. Plant Mol. Biol. (2004) 54(2):157-174.
8. VERMA NK, DEY CS: RNA-mediated gene silencing: mechanisms and its therapeutic applications. J. Clin. Pharm. Ther. (2004) 29(5):395-404.

9. TIJSTERMAN M, PLASTERK RH: Dicers at RISC; the mechanism of RNAi. Cell (2004) 117(1):1-3.

- Overview of the molecular players involved in RNAi.

10. OKAMURA K, ISHIZUKA A, SIOMI H, SIOMI MC: Distinct roles for Argonaute proteins in small RNA-directed RNA cleavage pathways. Genes Dev. (2004) 18(14):1655-1666.

11. PELLINO JL, SONTHEIMER EJ: R2D2 leads the silencing trigger to mRNA's death star. Cell (2003) 115(2):132-133.

12. MARTINEZ J, PATKANIOWSKA A, URLAUB H, LUHRMANN R, TUSCHL T: Single-stranded antisense siRNAs guide target RNA cleavage in RNAi. Cell (2002) 110(5):563-574.

13. KAWASAKI H, TAIRA K: Induction of DNA methylation and gene silencing by short interfering RNAs in human cells. Nature (2004) 431(7005):211-217.

14. MORRIS KV, CHAN SW, JACOBSEN SE, LOONEY DJ: Small interfering RNA-induced transcriptional gene silencing in human cells. Science (2004) 305(5688):1289-1292.

15. SAUNDERS LR, BARBER GN: The dsRNA binding protein family: critical roles, diverse cellular functions. FASEB J. (2003) 17(9):961-983.

16. SLEDZ CA, WILLIAMS BR: RNA interference and double-stranded-RNAactivated pathways. Biochem. Soc. Trans. (2004) 32(Pt 6):952-956.
17. PERSENGIEV SP, ZHU X, GREEN MR: Nonspecific, concentration-dependent stimulation and repression of mammalian gene expression by small interfering RNAs (siRNAs). RNA (2004) 10(1):12-18.

18. SLEDZ CA, HOLKO M, DE VEER MJ, SILVERMAN RH, WILLIAMS BR:

Activation of the interferon system by shortinterfering RNAs. Nat. Cell Biol. (2003) 5(9):834-839.

19. TIJSTERMAN M, MAY RC, SIMMER F, OKIHARA KL, PLASTERK RH: Genes required for systemic RNA interference in Caenorhabditis elegans. Curr. Biol. (2004) 14(2):111-116.

20. FEINBERG EH, HUNTER CP: Transport of dsRNA into cells by the transmembrane protein SID-1. Science (2003) 301(5639):1545-1547.

21. WINSTON WM, MOLODOWITCH C, HUNTER CP: Systemic RNAi in C. elegans requires the putative transmembrane protein SID-1. Science (2002) 295(5564):2456-2459.

22. SIJEN T, FLEENOR J, SIMMER F et al:: On the role of RNA amplification in dsRNA-triggered gene silencing. Cell (2001) 107(4):465-476.

23. VAN ROESSEL P, BRAND AH: Spreading silence with Sid. Genome Biol. (2004) 5(2):208.

24. MAINE EM: RNAi As a tool for understanding germline development in Caenorhabditis elegans: uses and cautions. Dev. Biol. (2001) 239(2):177-189.

25. BARGMANN CI: High-throughput reverse genetics: RNAi screens in Caenorhabditis elegans. Genome Biol. (2001) 2(2):REVIEWS1005. 
26. TIMMONS L, COURT DL, FIRE A: Ingestion of bacterially expressed dsRNAs can produce specific and potent genetic interference in Caenorhabditis elegans. Gene (2001) 263(1-2):103-112.

27. TIMMONS L, FIRE A: Specific interference by ingested dsRNA. Nature (1998) 395(6705):854.

28. DEVERAUX QL, AZA-BLANC P, WAGNER KW et al: : Exposing oncogenic dependencies for cancer drug target discovery and validation using RNAi. Semin. Cancer Biol. (2003) 13(4):293-300.

29. MOUSSES S, CAPLEN NJ, CORNELISON R et al.: RNAi microarray analysis in cultured mammalian cells. Genome Res. (2003) 13(10):2341-2347.

30. ARTS GJ, LANGEMEIJER E, TISSINGH R et al: : Adenoviral vectors expressing siRNAs for discovery and validation of gene function. Genome Res. (2003) 13(10):2325-2332.

31. DORSETT Y, TUSCHL T: siRNAs: applications in functional genomics and potential as therapeutics. Nat. Rev. Drug Discov. (2004) 3(4):318-329.

-. Review detailing the possible uses of siRNA.

32. DEVROE E, SILVER PA: Therapeutic potential of retroviral RNAi vectors. Expert Opin. Biol. Ther. (2004) 4(3):319-327.

33. LU PY, XIE FY, WOODLE MC: siRNAmediated antitumorigenesis for drug target validation and therapeutics. Curr. Opin. Mol. Ther. (2003) 5(3):225-234.

34. KRANENBURG O, GEBBINK MF, VOEST EE: Stimulation of angiogenesis by Ras proteins. Biochim. Biophys. Acta (2004) 1654(1):23-37.

35. MOUSA SA, MOUSA AS: Angiogenesis inhibitors: current $\&$ future directions. Curr. Pharm. Des. (2004) 10(1):1-9.

36. PAROO Z, COREY DR: Challenges for RNAi in vivo. Trends Biotech. (2004) 22(8):390-394.

37. GILMORE IR, FOX SP, HOLLINS AJ, SOHAIL M, AKHTAR S: The design and exogenous delivery of siRNA for posttranscriptional gene silencing. J. Drug Target. (2004) 12(6):315-340.

38. WALL NR, SHI Y: Small RNA: can RNA interference be exploited for therapy? Lancet (2003) 362(9393):1401-1403.

39. GRIFFIOEN AW, MOLEMA G: Angiogenesis: potentials for pharmacologic intervention in the treatment of cancer, cardiovascular diseases, and chronic inflammation. Pharmacol. Rev. (2000) 52(2):237-268.

- Review on the potential of angiogenesis targeted therapies.

40. SCHLINGEMANN RO: Role of growth factors and the wound healing response in age-related macular degeneration. Graefes Arch. Clin. Exp. Ophthalmol. (2004) 242(1):91-101.

41. GRANT MB, AFZAL A, SPOERRI P et al: : The role of growth factors in the pathogenesis of diabetic retinopathy. Expert Opin. Investig. Drugs (2004) 13(10):1275-1293.

42. MIDDLETON J, AMERICH L, GAYON R et al: : Endothelial cell phenotypes in the rheumatoid synovium: activated, angiogenic, apoptotic and leaky. Arthritis Res. Ther. (2004) 6(2):60-72.

43. BIAN XW, CHEN JH, JIANG XF et al.: Angiogenesis as an immunopharmacologic target in inflammation and cancer. Int. Immunopharmacol. (2004) 4(12):1537-1547.

44. SATO Y: Molecular diagnosis of tumor angiogenesis and anti-angiogenic cancer therapy. Int. J. Clin. Oncol. (2003) 8(4):200-206.

45. SUHARDJA A, HOFFMAN H: Role of growth factors and their receptors in proliferation of microvascular endothelial cells. Microsc. Res. Tech. (2003) 60(1):70-75.

46. TSOPANOGLOU NE, MARAGOUDAKIS ME: Role of thrombin in angiogenesis and tumor progression. Semin. Thromb. Hemost. (2004) 30(1):63-69.

47. JIN H, VARNER J: Integrins: roles in cancer development and as treatment targets. Br. J. Cancer (2004) 90(3):561-565.

48. SEIKI M, MORI H, KAJITA M, UEKITA T, ITOH Y: Membrane-type 1 matrix metalloproteinase and cell migration. Biochem. Soc. Symp. (2003) (70):253-262.

49. GERHARDT H, BETSHOLTZ C: Endothelial-pericyte interactions in angiogenesis. Cell Tissue Res. (2003) 314(1):15-23.

50. SCAVELLI C, WEBER E, AGLIANO M et al: Lymphatics at the crossroads of angiogenesis and lymphangiogenesis. J. Anat. (2004) 204(6):433-449.

51. ALITALO K, CARMELIET P: Molecular mechanisms of lymphangiogenesis in health and disease. Cancer Cell (2002) 1(3):219-227.
52. LOHELA M, SAARISTO A, VEIKKOLA T, ALITALO K: Lymphangiogenic growth factors, receptors and therapies. Thromb. Haemost. (2003) 90(2):167-184.

53. SHU X, WU W, MOSTELLER RD, BROEK D: Sphingosine kinase mediates vascular endothelial growth factor-induced activation of ras and mitogen-activated protein kinases. Mol. Cell. Biol. (2002) 22(22):7758-7768.

54. PETROVIC N, BHAGWAT SV, RATZAN WJ, OSTROWSKI MC, SHAPIRO LH: CD13/APN transcription is induced by RAS/MAPK-mediated phosphorylation of Ets-2 in activated endothelial cells. J. Biol. Chem. (2003) 278(49):49358-49368.

55. SOWTER HM, RAVAL RR, MOORE JW, RATCLIFFE PJ, HARRIS AL:

Predominant role of hypoxia-inducible transcription factor (Hif)-1alpha versus Hif-2alpha in regulation of the transcriptional response to hypoxia. Cancer Res. (2003) 63(19):6130-6134.

56. ZHANG L, YANG N, MOHAMED-HADLEY A, RUBIN SC, COUKOS G: Vector-based RNAi, a novel tool for isoform-specific knock-down of VEGF and anti-angiogenesis gene therapy of cancer. Biochem. Biophys. Res. Commun. (2003) 303(4):1169-1178.

57. MAZZANTI CM, TANDLE A, LORANG D et al: : Early genetic mechanisms underlying the inhibitory effects of endostatin and fumagillin on human endothelial cells. Genome Res. (2004) 14(8):1585-1593.

58. NAIK MU, VUPPALANCHI D, NAIK UP: Essential role of junctional adhesion molecule-1 in basic fibroblast growth factor-induced endothelial cell migration. Arterioscler. Thromb. Vasc. Biol. (2003) 23(12):2165-2171.

59. NAGASHIMA K, ENDO A, OGITA H et al: : Adaptor protein Crk is required for ephrin-B1-induced membrane ruffling and focal complex assembly of human aortic endothelial cells. Mol. Biol. Cell (2002) 13(12):4231-4242.

60. SULLIVAN DC, HUMINIECKI L, MOORE JW et al: : EndoPDI, a novel protein-disulfide isomerase-like protein that is preferentially expressed in endothelial cells acts as a stress survival factor. J. Biol. Chem. (2003) 278(47):47079-47088.

61. BALDANZI G, MITOLA S, CUTRUPI S et al: Activation of diacylglycerol kinase 
alpha is required for VEGF-induced angiogenic signaling in vitro. Oncogene (2004) 23(28):4828-4838.

62. BRUHL T, URBICH C, AICHER D et al: Homeobox A9 transcriptionally regulates the EphB4 receptor to modulate endothelial cell migration and tube formation. Circ. Res. (2004) 94(6):743-751.

63. ZHOU X, STUART A, DETTIN LE et al: Desmoplakin is required for microvascular tube formation in culture. J. Cell Sci. (2004) 117(Pt 15):3129-3140.

64. CONRAD C, ERFLE H, WARNAT P et al: Automatic identification of subcellular phenotypes on human cell arrays. Genome Res. (2004) 14(6):1130-1136.

65. SILVA JM, MIZUNO H, BRADY A, LUCITO R, HANNON GJ: RNA interference microarrays: high-throughput loss-of-function genetics in mammalian cells. Proc. Natl. Acad. Sci. USA (2004) 101(17):6548-6552.

66. SUGIMOTO A: High-throughput RNAi in Caenorhabditis elegans: genome-wide screens and functional genomics. Differentiation (2004) 72(2-3):81-91.

67. SHIBUYA M: VEGF-receptor inhibitors for anti-angiogenesis. Nippon Yakurigaku Zasshi (2003) 122(6):498-503.

68. ZACHARY I: VEGF signalling: integration and multi-tasking in endothelial cell biology. Biochem. Soc. Trans. (2003) 31(Pt 6):1171-1177.

69. HARRY LE, PALEOLOG EM: From the cradle to the clinic: VEGF in developmental, physiological, and pathological angiogenesis. Birth Defects Res. Part C Embryo Today (2003) 69(4):363-374.

70. UEDA Y, YAMAGISHI T, IKEYA $\mathrm{H}$ et al.: VGA1155, a novel binding antagonist of VEGF, inhibits angiogenesis in vitro and in vivo. Anti-Cancer Res. (2004) 24(5A):3009-3017.

71. UEDA Y, YAMAGISHI T, SAMATA K et al: : A novel low molecular weight VEGF receptor-binding antagonist, VGA1102, inhibits the function of VEGF and in vivo tumor growth. Cancer Chemother. Pharmacol. (2004) 54(1):16-24.

72. INAI T, MANCUSO M, HASHIZUME H et al:: Inhibition of vascular endothelial growth factor (VEGF) signaling in cancer causes loss of endothelial fenestrations, regression of tumor vessels, and appearance of basement membrane ghosts. Am. J. Pathol. (2004) 165(1):35-52.

73. FILLEUR S, COURTIN A, AIT-SI-ALI S et al: SiRNA-mediated inhibition of vascular endothelial growth factor severely limits tumor resistance to antiangiogenic thrombospondin-1 and slows tumor vascularization and growth. Cancer Res. (2003) 63(14):3919-3922.

74. DUXBURY MS, MATROS E, ITO H et al: Systemic siRNA-mediated gene silencing: a new approach to targeted therapy of cancer. Ann. Surg. (2004) 240(4):667-674; discussion 675-666.

75. AHARINEJAD S, PAULUS P, SIOUD M et al: : Colony-stimulating factor-1 blockade by antisense oligonucleotides and small interfering RNAs suppresses growth of human mammary tumor xenografts in mice. Cancer Res. (2004) 64(15):5378-5384.

76. LAKKA SS, GONDI CS, YANAMANDRA $\mathrm{N}$ et al: : Inhibition of cathepsin B and MMP-9 gene expression in glioblastoma cell line via RNA interference reduces tumor cell invasion, tumor growth and angiogenesis. Oncogene (2004) 23(27):4681-4689.

77. CHAE SS, PAIK JH, FURNEAUX H, HLA T: Requirement for sphingosine 1phosphate receptor- 1 in tumor angiogenesis demonstrated by in vivo RNA interference. J. Clin. Invest. (2004) 114(8):1082-1089.

78. TAKEI Y, KADOMATSU K, YUZAWA Y, MATSUO S, MURAMATSU T: A small interfering RNA targeting vascular endothelial growth factor as cancer therapeutics. Cancer Res. (2004) 64(10):3365-3370.

79. SCHIFFELERS RM, ANSARI A, XU J et al: : Cancer siRNA therapy by tumor selective delivery with ligand-targeted sterically stabilized nanoparticle. Nucleic Acids Res. (2004) 32(19):e149.

80. KIM B, TANG Q, BISWAS PS et al.: Inhibition of ocular angiogenesis by siRNA targeting vascular endothelial growth factorpathway genes; therapeutic strategy for herpetic stromal keratitis. Am. J. Pathol. (2004) 165(6):2177-2185.

81. JACKSON AL, BARTZ SR, SCHELTER J et al: : Expression profiling reveals off-target gene regulation by RNAi. Nat. Biotechnol. (2003) 21(6):635-637.

- One of the first reports questioning the specificity of RNAi.
82. SAXENA S, JONSSON ZO, DUTTA A: Small RNAs with imperfect match to endogenous mRNA repress translation. Implications for off-target activity of small inhibitory RNA in mammalian cells. J. Biol. Chem. (2003) 278(45):44312-44319.

83. SNOVE O JR, HOLEN T: Many commonly used siRNAs risk off-target activity. Biochem. Biophys. Res. Commun. (2004) 319(1):256-263.

84. SCACHERI PC, ROZENBLATT-ROSEN O, CAPLEN NJ et al: : Short interfering RNAs can induce unexpected and divergent changes in the levels of untargeted proteins in mammalian cells. Proc. Natl. Acad. Sci. USA (2004) 101(7):1892-1897.

85. ABBAS-TERKI T, BLANCO-BOSE W, DEGLON N, PRALONG W, AEBISCHER P: Lentiviral-mediated RNA interference. Hum. Gene Ther. (2002) 13(18):2197-2201.

86. DEVROE E, SILVER PA: Retrovirusdelivered siRNA. BMC Biotechnol. (2002) 2(1):15.

87. TOMAR RS, MATTA H, CHAUDHARY PM: Use of adenoassociated viral vector for delivery of small interfering RNA. Oncogene (2003) 22(36):5712-5715.

88. CHIU YL, RANA TM: siRNA function in RNAi: a chemical modification analysis. $R N A$ (2003) 9(9):1034-1048.

89. AMARZGUIOUI M, HOLEN T, BABAIE E, PRYDZ H: Tolerance for mutations and chemical modifications in a siRNA. Nucleic Acids Res. (2003) 31(2):589-595.

90. LAYZER JM, MCCAFFREY AP, TANNER AK et al: In vivo activity of nuclease-resistant siRNAs. RNA (2004) 10(5):766-771.

\section{Affiliation}

Raymond M Schiffelers ${ }^{\dagger}$ Inge van Rooy \& Gert Storm

${ }^{\dagger}$ Author for correspondence

Utrecht Institute for Pharmaceutical Sciences, Room Z 735 A, Department of Pharmaceutics, Utrecht University, PO Box 80.082, 3508 TB, Utrecht, The Netherlands Tel: +31 30 2539392; Fax: +31 30 2517839; E-mail: R.M.Schiffelers@pharm.uu.nl 